\title{
Hepatitis E Virus Seroprevalence Rate in HIV-Infected Patients in Germany: A Comparison of Two Commercial Assays
}

\author{
Sven Pischke ${ }^{a, b} \quad$ Carolynne Schwarze-Zander ${ }^{c}$ Birgit Bremer ${ }^{a}$ \\ Patrick Lehmann $^{\text {a }}$ Steffen B. Wiegand ${ }^{a}$ Anett Gisa ${ }^{a}$ \\ Patrick Behrendt $^{\mathrm{a}}$ Christian P. Strassburg ${ }^{c}$ Michael P. Manns ${ }^{\mathrm{a}}$ \\ Heiner Wedemeyer ${ }^{\mathrm{a}} \quad$ Jürgen K. Rockstroh ${ }^{\mathrm{c}}$ \\ ${ }^{a}$ Department of Gastroenterology, Hepatology and Endocrinology, Hannover Medical School, Hannover, \\ ${ }^{b}$ MVZ für Lebertransplantation, Gastroenterology, University Hospital Hamburg Eppendorf, Hamburg, and \\ 'Department of Gastroenterology, University Hospital of Bonn, Bonn, Germany
}

\section{Key Words}

HIV · Hepatitis E virus · Seroprevalence · Wantai assay ·

MP assay

\begin{abstract}
Objectives: Cases of chronic hepatitis E virus (HEV) infection have been described in HIV-infected patients. There are several commercial anti-HEV assays, but anti-HEV seroprevalence rates differ largely depending on the assay used. The aim of this study was to (1) compare two commercial antiHEV assays in a German cohort of HIV-positive individuals, and (2) determine whether HEV takes chronic courses in controlled HIV infection. Methods: 246 HIV patients were tested for both HEV RNA and HEV antibodies. All patients received antiretroviral therapy, if this was indicated, according to European guidelines. All but 19 individuals had CD4+ counts above $200 / \mu \mathrm{l}$. Anti-HEV lgG was determined by two independent commercial assays (Wantai and MP). Results: None of the patients tested HEV RNA positive. Anti-HEV IgG was detected more frequently by the Wantai assay (26\%) than the MP assay $(1.6 \%, p<0.001)$. Patients born in Europe test-
\end{abstract}

ed more frequently positive for anti-HEV ( $p=0.047$ ) than individuals from other regions. Increasing age but not CD4 count correlated with a higher likelihood of anti-HEV positivity $(R=0.313, p<0.001)$. Conclusions: About one quarter of HIV-infected patients show evidence of previous HEV contact. The risk of developing chronic HEV infection is very low in individuals receiving appropriate antiretroviral therapy. The large variability in HEV seroprevalence rates determined by different assays requires consideration for the diagnostic workup of HIV patients.

๑) 2015 S. Karger AG, Basel

\section{Introduction}

Hepatitis E virus (HEV) infection has been described to lead to chronic inflammation of the liver in immunosuppressed individuals, including transplant recipients and HIV-infected patients [1-3]. Studies from Argentina

Heiner Wedemeyer and Jürgen K. Rockstroh contributed equally and share senior authorship.

\section{KARGER 125}

(c) 2015 S. Karger AG, Base

$0300-5526 / 15 / 0585-0283 \$ 39.50 / 0$

E-Mail karger@karger.com

www.karger.com/int
Prof. Dr. Heiner Wedemeyer

Department of Gastroenterology, Hepatology and Endocrinology

Medizinische Hochschule Hannover, Carl-Neuberg Strasse 1

DE-30625 Hannover (Germany)

E-Mail Wedemeyer.Heiner@mh-hannover.de 
[4] and Russia [5] performed in the 1990s suggested that $\mathrm{HIV}$-infected patients may have an increased anti-HEV seroprevalence rate in comparison to healthy controls, indicating a higher exposure of HIV-infected patients to HEV. In contrast, a recent study from England showed no difference in anti-EV seroprevalence rate in $138 \mathrm{HIV}$ infected patients in comparison to 464 healthy controls [6].

However, more advanced stages of HIV infection have been associated with a strongly increased frequency of anti-HEV [5]. However, specific risk factors for contact with HEV within the population of HIV-infected patients are largely unknown. While consumption of pork meat and blood products have been demonstrated to be possible sources of HEV transmission in Europe [7], sexual behavior or drug abuse have been discussed but are not accepted risk factors $[4,5]$.

Various assays to detect anti-HEV have been described in recent years. Striking differences in sensitivities and specificities between the assays have become evident [8]. Recently, a study comparing anti-HEV seroprevalence rates in liver and kidney transplant recipients demonstrated a huge gap between two commercially available assays: the Adaltis assay determined a seroprevalence rate of $11 \%$ in this cohort, while the Wantai assay tested positive in $31 \%$ of patients [9]. This study suggested that the Wantai assay is more sensitive to detect anti-HEV IgG antibodies, even though the small size $(n=64)$ represented a relevant limitation of this study. Furthermore, a comparative study on 88 immunosuppressed individuals indicated that the Wantai assay had a sensitivity superior to the MP assay [10].

Different anti-HEV assays have not yet been studied specifically in the context of HIV infection. In particular, nothing is known about the performance of the Wantai assay in HIV-infected patients.

The aims of this study were therefore (1) to identify the anti-HEV seroprevalence rate in HIV-infected patients in Germany by comparison of the Wantai assay with the MP assay, (2) to identify risk factors for HEV contact by analyzing patient characteristics and (3) to determine if HEV causes chronic hepatitis E under controlled HIV infection.

\section{Patients and Methods}

In a retrospective study, 246 blood samples from HIV-infected patients were tested for anti-HEV IgG antibodies and HEV RNA. All patients were recruited at the University Hospital of Bonn between June 2009 and December 2009, and samples were stored at $-20^{\circ}$ until testing in November 2012. Importantly, all samples were tested both for HEV IgG and RNA irrespective of the HEV serostatus. This study was approved by the local Ethics Committee of Hannover Medical School and the University Hospital of Bonn. Patients gave informed consent for collection and testing of their blood.

\section{Anti-HEV and HEV RNA Testing}

Anti-HEV IgG antibodies were tested both with the MP Diagnostic Assay (MP Biomedicals, formerly Genelabs Diagnostics, Singapore) and with the Wantai Diagnostic Assay (Beijing, China) according to the manufacturer's instructions.

The MP assay detects antibodies against a mixture of recombinant peptides specified by ORF2 and ORF3. This assay was developed based on two different strains of HEV: one genotype 1 strain from Burma and a genotype 2 strain from Mexico. The Wantai assay was developed based on a recombinant peptide corresponding to amino acid residues 396-606 of the major structural protein specified by ORF2 of genotype 4 from China [11].

HEV RNA testing was performed using nested PCR. The following primer sequences were used, as described previously [12]:

1 PCR HE 361F GCR GTG GTT TCT GGG GTG AC HE 364R CTG GGM YTG GTC DCG CCA AG

2 PCR HE 366F GYT GAT TCT CAG CCC TTC GC HE 363R GMY TGG TCD CGC CAA GHG GA1

\section{Statistics}

Data for different patient groups are presented as means, ranges and standard deviations. A comparison of continuous and categorical data between groups was performed using the $\chi^{2}$ test. Comparison of quantitative data between groups was performed using the Mann-Whitney test. Correlation was calculated using the Pearson method. A p value $<0.05$ was considered significant.

\section{Results}

Most of patients received antiretroviral therapy according to European guidelines at the time of the respective blood drawing, while 31 patients (13\%) were not treated. HIV viral loads were below the limit of detection in $76 \%$ of the patients $(n=186)$ and CD4 T cell counts were below $200 \mu$ in $7.7 \%$ of the patients $(n=19)$. Patient characteristics are shown in detail in table 1.

Anti-HEV antibodies tested positive in $4(1.6 \%)$ and $63(26 \%)$ patients with the MP assay and the Wantai assay, respectively (fig. $1 ; \mathrm{p}<0.001$ ). In 4 additional patients the Wantai test result gave a borderline signal-to-cutoff ratio (1.6\%). Two of the 4 patients who tested positive with the MP assay also tested positive with the Wantai assay, while 2 tested negative.

Wantai anti-HEV-positive patients did not differ significantly from negative patients regarding clinical characteristics such as CD4 count, ALT and AST levels, or coinfections with other hepatitis viruses. However, there 
Table 1. Characteristics of the study population

\begin{tabular}{lccc}
\hline Characteristics & $\begin{array}{l}\text { Patients overall } \\
(\mathrm{n}=246)\end{array}$ & $\begin{array}{l}\text { MP-positive patients } \\
(\mathrm{n}=4)\end{array}$ & $\begin{array}{l}\text { Wantai-positive patients } \\
(\mathrm{n}=63)\end{array}$ \\
\hline Age, years $^{1}$ & $46(18-76,11)$ & $42(35-50,7)$ & $52(27-73,11)$ \\
ALT $^{1}$ & $33(9-212,26)$ & $38(15-53,17)$ & $33(14-163,25)$ \\
HIV load $^{2}$ & $0(0-841,414,65,163)$ & $0(0-106,61)$ & $0(0-52,009,11,364)$ \\
CD4 count & $532(27-1,873,278)$ & $406(108-831,325)$ & $491(27-1,108,229)$ \\
Detectable HIV & $59(24)$ & $1(25)$ & $14(22)$ \\
CD4 count $<200 / \mu \mathrm{l}$ & $19(8)$ & $1(25)$ & $6(10)$ \\
Origin Africa & $30(12)$ & $0(0)$ & $3(5)$ \\
Origin America & $1(<1)$ & $0(0)$ & $0(0)$ \\
Origin Asia & $8(3)$ & $0(0)$ & $1(2)$ \\
Origin Europe & $210(85)$ & $4(100)$ & $7(94)$ \\
Chronic HCV infection & $27(11)$ & $1(25)$ & $2(3)$ \\
Chronic HBV infection & $10(4)$ & $(0)$ & (1) \\
\hline
\end{tabular}

Values are $\mathrm{n}(\%)$ unless otherwise indicated. ${ }^{1}$ Mean (range, SD). ${ }^{2}$ Median (range, SD).

were differences between the factors 'age' and 'country of origin' between positive and negative individuals (table 1). Increasing age correlated significantly with a higher likelihood of anti-HEV positivity in the Wantai assay $(\mathrm{R}=0.313, \mathrm{p}<0.001)$.

All 4 patients who tested positive with the MP assay and 59 of 63 (94\%) patients who tested positive with the Wantai assay were from Europe, while only 147 of 179 Wantai-negative patients (82\%) were born in European countries $(\mathrm{p}=0.047)$. Out of the patients with a CD4 count below $200 \mu \mathrm{l}, 1$ out of 19 tested positive with the MP assay (5\%) and 6 out of 19 tested positive with the Wantai assay (32\%). Out of the patients viremic for HIV , 1 out of 59 (2\%) tested positive with the MP assay and 14 out of $59(24 \%)$ tested positive with the Wantai assay (table 1). All 246 samples tested HEV RNA negative, excluding cases of chronic hepatitis $\mathrm{E}$ in this cohort.

\section{Discussion}

We demonstrated a remarkable discrepancy of seroprevalence rates of anti-HEV antibodies in HIV-infected patients determined by two commercially available assays. In comparison to previously described seroprevalence rates in the healthy population in Germany determined by the same assays, HIV-infected patients do not have an increased seroprevalence [8]. While the MP assay showed very low frequencies of anti-HEV,

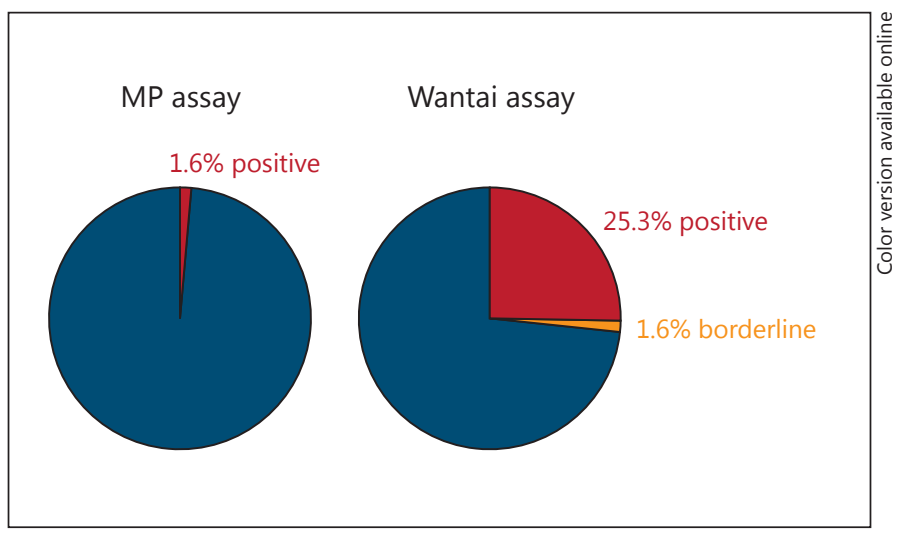

Fig. 1. Comparison of anti-HEV seroprevalence rates determined by two different assays.

more than one quarter of the patients tested anti-HEV positive with the Wantai assay, which is frequently used by various groups in different countries and has been shown to have a high sensitivity in immunosuppressed and immunocompromised individuals, such as transplant recipients $[8,9]$. It is unlikely that the difference in these seroprevalence rates is caused by false-positive results using the Wantai assay, as a high specificity of this assay has been described [13]. However, a low specificity of this assay could not been ruled out completely and is one possible explanation for the large discrepancy in determined seroprevalence rates, but a superior 
sensitivity of the Wantai assay is the most likely explanation.

The observation that 2 of the 4 patients who tested positive with the MP assay tested negative with the Wantai assay indicates that the MP assay might have tested these patients false positive, thus demonstrating a lower specificity of this assay. The alternative explanation that the Wantai assay was false negative in these 2 patients is unlikely due to the high sensitivity of this assay [8-10].

Overall, the findings presented here for HIV-infected patients are well in line with previously published studies examining anti-HEV seroprevalence rates determined by two independent assays in transplant recipients and immunocompetent individuals $[9,10]$.

Determination of previous HEV exposure in special patient groups is essential to identify risk groups, risk factors and sources of infection. This is particularly important among HIV-infected patients in whom HEV infection may progress to chronic infection associated with development of advanced liver disease. Fortunately, none of the patients in the present cohort was chronically infected with HEV. Thus, this study and previous investigations confirmed that chronic HEV infection in $\mathrm{HIV}$-infected patients is rather rare. However, chronic hepatitis E might be more frequent in untreated HIVinfected patients, in particular those with a strongly decreased immune system [14].

Recently, a study comparing the MP assay and the Wantai assay in 200 healthy people in Germany determined seroprevalence rates of 4.5 and $29.5 \%$, respectively [8]; therefore, the anti-HEV seroprevalence rate in HIVinfected patients in Germany does not seem to be increased in comparison to healthy controls. This finding is in line with a previous study on the relevance of HEV in
HIV-infected patients in Germany which was also not able to find an increased anti-HEV seroprevalence rate in $\mathrm{HIV}$-infected patients using the an anti-HEV assay, based on the same antigens as the present MP assay [15].

A second potentially important aspect of the present study is the finding that most of those patients testing positive for anti-HEV are from Europe. Migrants from tropical countries do not show a higher anti-HEV seroprevalence rate in this study, which highlights the importance of autochthonous HEV infections in Europe in contrast to imported infection. This finding is in line with a previous case control study indicating that most acute HEV infections in Germany have been acquired autochthonously [16]. However, the exact time point of seroconversion, indicating contact to HEV, could not be described in this study, and thus it is possible that the migrants acquired their HEV infection in Germany. As data on the time point are lacking, we do not want to overestimate this difference.

While HEV genotypes 1 and 2 are limited to developing tropical countries, genotypes 3 and 4 are present in industrialized nations. Even though there are more observed HEV infections in developing countries, our study does not demonstrate a higher HEV seroprevalence marking a higher HEV exposition in migrants from tropical regions in contrast to patients from Germany. These data support the hypothesis that most genotype 3 infections occur clinically silently [17].

In conclusion, about one quarter of HIV-infected patients in Germany have evidence of previous contact to $\mathrm{HEV}$. Still, the risk of developing chronic hepatitis $\mathrm{E}$ is very low in individuals receiving appropriate antiretroviral therapy. The large variability in HEV seroprevalence rates determined by different assays requires consideration for the diagnostic workup of HIV patients.

\section{References}

1 Wedemeyer H, Pischke S, Manns MP: Pathogenesis and treatment of hepatitis $\mathrm{E}$ virus infection. Gastroenterology 2012;142:13881397.e1.

2 Dalton HR, Bendall RP, Keane FE, Tedder RS, Ijaz S: Persistent carriage of hepatitis E virus in patients with HIV infection. N Engl J Med 2009;361:1025-1027.

3 Kenfak-Foguena A, Schöni-Affolter F, Bürgisser $\mathrm{P}$, Witteck A, Darling KE, Kovari $\mathrm{H}$, Kaiser L, et al: Hepatitis E virus seroprevalence and chronic infections in patients with HIV, Switzerland. Emerg Infect Dis 2011;17: 1074-1078.
4 Fainboim H, Gonzalez J, Fassio E, Martinez A, Otegui L, Eposto M, Cahn P, et al: Prevalence of hepatitis viruses in an anti-human immunodeficiency virus-positive population from Argentina. A multicentre study. J Viral Hepat 1999;6:53-57.

5 Balayan MS, Fedorova OE, Mikhailov MI, Rytick PG, Eremin VF, Danilova TI, Shevelev BI, et al: Antibody to hepatitis E virus in HIVinfected individuals and AIDS patients. J Viral Hepat 1997;4:279-283.
6 Keane F, Gompels M, Bendall R, Drayton R, Jennings L, Black J, Baragwanath G, et al: Hepatitis E virus coinfection in patients with HIV infection. HIV Med 2012;13:83-88.

7 Pischke S, Behrendt P, Bock CT, Jilg W, Manns MP, Wedemeyer H: Hepatitis E in Germany - an under-reported infectious disease. Dtsch Arztebl Int 2014;111:577-583.

8 Wenzel JJ, Preiss J, Schemmerer M, Huber B, Jilg W: Test performance characteristics of Anti-HEV IgG assays strongly influence hepatitis E seroprevalence estimates. J Infect Dis 2013;207:497-500. 
9 Rossi-Tamisier M, Moal V, Gerolami R, Colson P: Discrepancy between anti-hepatitis E virus immunoglobulin $G$ prevalence assessed by two assays in kidney and liver transplant recipients. J Clin Virol 2013;56:62-64.

10 Pas SD, Streefkerk RH, Pronk M, de Man RA, Beersma MF, Osterhaus AD, van der Eijk AA: Diagnostic performance of selected commercial HEV IgM and IgG ELISAs for immunocompromised and immunocompetent patients. J Clin Virol 2013;58:629-634.

11 Park HK, Jeong SH, Kim JW, Woo BH, Lee DH, Kim HY, Ahn S: Seroprevalence of antihepatitis E virus (HEV) in a Korean population: comparison of two commercial antiHEV assays. BMC Infect Dis 2012;12:142.
12 Pischke S, Stiefel P, Franz B, Bremer B, Suneetha PV, Heim A, Ganzenmueller T, et al: Chronic hepatitis $\mathrm{E}$ in heart transplant recipients. Am J Transplant 2012;12:31283133.

13 Khan A, Tanaka Y, Kurbanov F, Elkady A, Abbas Z, Azam Z, Subhan A, et al: Investigating an outbreak of acute viral hepatitis caused by hepatitis E virus variants in Karachi, South Pakistan. J Med Virol 2011;83:622-629.

14 Dalton HR, Keane FE, Bendall R, Mathew J, Ijaz S: Treatment of chronic hepatitis $\mathrm{E}$ in a patient with HIV infection. Ann Intern Med 2011;155:479-480.
15 Pischke S, Ho H, Urbanek F, Meyer-Olsen D, Suneetha PV, Manns MP, Stoll M, et al: Hepatitis $\mathrm{E}$ in HIV-positive patients in a low-endemic country. J Viral Hepat 2010;17:598599

16 Wichmann O, Schimanski S, Koch J, Kohler M, Rothe C, Plentz A, Jilg W, et al: Phylogenetic and case-control study on hepatitis $\mathrm{E}$ virus infection in Germany. J Infect Dis 2008; 198:1732-1741.

17 Shata MT, Navaneethan U: The mystery of hepatitis E seroprevalence in developed countries: is there subclinical infection due to hepatitis E virus? Clin Infect Dis 2008;47:10321034. 